\title{
Increased MALAT1 expression contributes to cisplatin resistance in non-small cell lung cancer
}

\author{
YONG CUI, GUANLONG LI, XIN ZHANG, FANGFANG DAI and RONGXIANG ZHANG
}

The Third Department of Oncology, Shouguang People's Hospital, Shouguang, Shandong 262700, P.R. China

Received October 17, 2017; Accepted April 4, 2018

DOI: $10.3892 / \mathrm{ol} .2018 .9293$

\begin{abstract}
Cisplatin-based chemotherapy is commonly used for the clinical treatment of patients with non-small cell lung cancer (NSCLC). However, the anti-tumor efficacy of cisplatin is limited by poor clinical response and the development of chemoresistance. At present, the underlying mechanism for cisplatin resistance remains unclear. In the present study, it was identified that metastasis-associated lung adenocarcinoma transcript 1 (MALAT1), a long non-coding RNA that has been demonstrated to function as an oncogene, was increased in tumor tissues from patients with cisplatin-resistant NSCLC. In addition, the MALAT1 level was increased in A549rCDDP cells compared with the parental A549 cells. Silencing of MALAT1 sensitized A549rCDDP cells to cisplatin treatment, while overexpression of MALAT1 in A549 cells decreased their sensitivity towards cisplatin. Through analysis of the gene expression in patient samples, a decrease in miR-145 and an increase in Kruppel-like factor 4 (KLF4) in tumor tissues compared with adjacent normal tissues was observed. A negative association between MALAT1 and miR-145 was also identified in A549 cells and A549rCDDP cells. Furthermore, reverse transcription quantitative polymerase chain reaction and western blotting identified that KLF4 was positively and negatively regulated by MALAT1 and miR-145, respectively. The direct regulatory association between MALAT1 and miR-145 and the target gene KLF4 was additionally confirmed using a luciferase reporter assay. Knockdown of MALAT1 reversed cisplatin resistance in A549rCDDP cells. Taken together, these data indicated that MALAT1 decreased the sensitivity of NSCLC to cisplatin via the regulation of miR-145 and KLF4.
\end{abstract}

Correspondence to: Dr Yong Cui, The Third Department of Oncology, Shouguang People's Hospital, 1233 Jiankang Street, Shouguang, Shandong 262700, P.R. China

E-mail: cuiyongsg@aliyun.com

Key words: metastasis-associated lung adenocarcinoma transcript 1 , microRNA-145, kruppel-like factor 4, cisplatin resistance, non-small cell lung cancer

\section{Introduction}

A total of $>85 \%$ lung cancer is classified as non-small cell lung cancer (NSCLC) (1). Although early diagnosis and novel therapeutic approaches have markedly prolonged the overall survival of patients with NSCLC, the survival rate for patients with drug resistance remains low (2).

Long non-coding RNAs (lncRNAs) are transcripts consisting of $>200$ nucleotides with no ability to code protein (3). Previous studies have indicated that lncRNAs were involved in the pathogenesis of various diseases, including cancer (4). Metastasis-associated lung adenocarcinoma transcript 1 (MALAT1) has been identified to regulate cancer cell proliferation, migration and invasion in lung, hepatocellular, ovarian and colorectal cancer (5-7). MALAT1 was also demonstrated to induce temozolomide resistance in glioblastoma (8). However, whether and how MALAT1 contributes to cisplatin resistance in NSCLC is unknown.

MicroRNAs (miRNAs) are small non-coding RNAs that function in RNA silencing through complementary binding. miR-145 is a well-known tumor suppressor that inhibits oncogene expression in numerous types of cancer $(9,10)$. Additionally, increased miR-145 expression levels increased the sensitivity of hepatocellular carcinoma cells to chemotherapy via the downregulation of Mothers against decapentaplegic homolog 3 (11). In lung cancer, miR-145 was suggested to inhibit cancer cell proliferation, migration and invasion, but its role in chemoresistance was not identified (12).

Cancer stem cells have drawn much attention for their pivotal role in promoting relapse and drug resistance, including cisplatin resistance (13). With the ability to maintain cancer cell stemness, Kruppel-like factor 4 (KLF4) serves as an oncogene in a number of types of cancer, and has been demonstrated to be associated with chemoresistance $(14,15)$. For example, the interaction between KLF4 and High mobility group box 1 conferred resistance in osteosarcoma cells to multiple chemotherapy agents, including cisplatin (16). In lung cancer, an increased expression of KLF4 was observed in high-grade NSCLC compared with low-grade disease (17). Increased Hox transcript antisense RNA and KLF4 levels were involved in cisplatin resistance in NSCLC (18), but the underlying mechanism remains unknown.

The present study aimed to explore whether and how MALAT1 may affect the sensitivity of NSCLC towards cisplatin. It was identified that MALAT1 and KLF4 levels were 
increased and miR-145 levels were decreased in tumor tissues from patients with cisplatin-refractory NSCLC compared with those from cisplatin-sensitive NSCLC patients. Silencing of MALAT1 sensitized A549rCDDP cells to cisplatin, while the overexpression of MALAT1 induced cisplatin resistance in A549 cells. Importantly, a direct regulatory association between MALAT1 and miR-145 and the target gene KLF4 was confirmed. Taken together, the present study highlighted the role of MALAT1 in the development of cisplatin-resistance in NSCLC.

\section{Materials and methods}

Patients. A total of 52 tumor tissue samples were collected from 31 patients with NSCLC for whom cisplatin-based chemotherapy was effective following surgery (patients who were cisplatin-sensitive), and 21 patients with NSCLC for whom cisplatin-based chemotherapy was ineffective following surgery (patients who were cisplatin-resistant). Histopathological types were assigned using WHO pathological staging criteria (19). All patients were administrated $100 \mathrm{mg} / \mathrm{m}^{2}$ cisplatin intravenously over 30 to $120 \mathrm{~min}$ on day 1 of the 28 day cycle. The total number of cycles administrated was one. The samples were obtained from Shouguang People's Hospital (Shouguang, China) from June 2012 to June 2015. The present study was approved by the Ethics Committee of Shouguang People's Hospital. All participants provided written informed consent prior to surgery. Patients did not receive radiotherapy or chemotherapy prior to surgery. The tissues were immediately frozen for protein and RNA extraction.

Cell culture and agents. The human NSCLC A549 and H1299 cell lines were purchased from American Type Culture Center (Manassas, VA, USA) and the A549 cisplatin-resistant A549rCDDP subline was obtained from the Cancer Hospital of Peking Union Medical College, Chinese Academy of Medical Sciences (Beijing, China). All cell lines were cultured in RPMI-1640 medium (Gibco; Thermo Fisher Scientific, Inc., Waltham, MA, USA) containing $10 \%$ fetal bovine serum (Hyclone; GE Healthcare Life Sciences, Logan, UT, USA). The medium for A549rCDDP cells was additionally supplemented with $2 \mathrm{mg} / \mathrm{l}$ cisplatin (Selleck Chemicals, Houston, TX, USA). All cell lines were cultivated in a humid incubator at $37^{\circ} \mathrm{C}$ with $5 \% \mathrm{CO}_{2}$. For cisplatin treatment, $1,2,5$ or $10 \mu \mathrm{M}$ of cisplatin was added into the culture medium of A549 cells or A549rCDDP cells for $48 \mathrm{~h}$, and then the cells were used for subsequent experimentation.

RNA extraction and reverse transcription quantitative polymerase chain reaction $(R T-q P C R)$. Total RNA was extracted from tissues and A549, H1299 and A549rCDDP cells using the miRNeasy Mini kit (QIAGEN, Hilden, Germany) according to the manufacturer's protocol. A Molony-Murine Leukemia Virus kit (Life Technologies; Thermo Fisher Scientific, Inc.) was used to synthesize cDNA. qPCR was performed with a CFX96 Touch ${ }^{\mathrm{TM}}$ system (Bio-Rad Laboratories, Inc., Hercules, CA, USA) using SYBR Premix Ex Taq (Takara Bio, Inc., Otsu, Japan). The thermocycling conditions for miR-145 and U6 were as follows: $95^{\circ} \mathrm{C}$ for $10 \mathrm{~min}, 40$ cycles of $95^{\circ} \mathrm{C}$ for $15 \mathrm{sec}$ and $60^{\circ} \mathrm{C}$ for $1 \mathrm{~min}$. The thermocycling conditions for IncRNA, mRNA and GAPDH were as follows: $95^{\circ} \mathrm{C}$ for $10 \mathrm{~min}$, followed by 40 cycles at $95^{\circ} \mathrm{C}$ for $15 \mathrm{sec}, 55^{\circ} \mathrm{C}$ for $30 \mathrm{sec}$ and $72^{\circ} \mathrm{C}$ for $30 \mathrm{sec}$. GAPDH was used as an internal control for lncRNA and mRNA, and U6 was applied as an internal control for miRNA. The relative expression levels were calculated using the $2^{-\Delta \Delta \mathrm{Cq}}$ method (20). The sequences for the primers were as follows: MALAT1 forward, AGACCTTGAAATCCAT; MALAT1 reverse, CTT CTGCTTCCTACTT; miR-145 forward, ACACTCCAGCTG GGGTCCAGTTTTCCCAGGA; miR-145 reverse, TGGTGT CGTGGAGTCG; KLF4 forward, GTCAGTTCATCTGAG CGGG; KLF4 reverse, AGAGTTCCCATCTCAAGGCA; GAPDH forward, AGAAGGCTGGGGCTCATTTG; GAPDH reverse, AGGGGCCATCCACAGTCTTC; U6 forward, CTC GCTTCGGCAGCACA; and U6 reverse, TGGTGTCGTGGA GTCG.

Western blotting. Protein was measured with a bicinchoninic acid kit (Sigma-Aldrich; Merck KGaA, Darmstadt, Germany) according to manufacturer's protocol. HRP-conjugated secondary antibodies for mouse (SA00001-1) and rabbit (SA00001-2) were purchased from Proteintech (Rosemont, IL). The antibody for GAPDH (catalog no. G8795, 1:10,000) was purchased from Sigma Aldrich; Merck KGaA. The KLF4 antibody (catalog no. 12173, 1:1,000) was purchased from Cell Signaling Technology, Inc. (Danvers, MA, USA). A549 cells were lysed in radioimmunoprecipitation assay lysis buffer (Beyotime Institute of Biotechnology, Haimen, China) containing protease inhibitor (Sigma Aldrich; Merck $\mathrm{KGaA}$ ). $20 \mu \mathrm{g}$ protein was separated by $8 \%$ SDS-PAGE and then transferred to a polyvinylidene fluoride membrane (EMD Millipore, Billerica, MA USA). Subsequent to blocking at room temperature in $5 \%$ non-fat milk for $30 \mathrm{~min}$, the membranes were incubated with the indicated primary antibodies overnight at $4^{\circ} \mathrm{C}$. The membranes were then incubated with a HRP-conjugated secondary antibody for $1 \mathrm{~h}$ at room temperature; anti-mouse antibody at 1:10,000 for GAPDH (catalog no. SA00001-1, Proteintech, Rosemont, IL), and anti-rabbit at 1:10,000 for KLF4 (catalog no. SA00001-2, Proteintech, Rosemont, IL). The membranes were developed using Pierce ECL Plus substrate (Thermo Fisher Scientific, Inc.). Images were captured with a densitometer (GS-700; Bio-Rad Laboratories, Inc.), analysis of protein expression was achieved using ImageJ software (Version $1.51 \mathrm{k}$, Rawak Software, Inc., Germany). GAPDH served as an internal control.

Cell viability assay. Cell viability was assessed using a Cell Counting Kit-8 (CCK-8; Dojindo Molecular Technologies, Kumamoto, Japan). 5,000 A549, H1299 or A549rCDDP cells were seeded in a 96-well plate with DMSO (Beijing Solarbio Science \& Technology Co., Ltd., Beijing, China) or 1, 2, 5 or $10 \mu \mathrm{M}$ of cisplatin and cultured at $37^{\circ} \mathrm{C}$ for $72 \mathrm{~h}$. Subsequently, $10 \mu \mathrm{l}$ CCK8 solution was added into each well and incubated at $37^{\circ} \mathrm{C}$ for $2 \mathrm{~h}$. The absorbance of each well at $450 \mathrm{~nm}$ was detected using a microplate reader (Bio-Rad Laboratories, Inc.) to measure cell viability.

Cell apoptosis assay. A cell apoptotic assay was performed using an Annexin-V/Dead Cell Apoptosis kit (Invitrogen; 
Thermo Fisher Scientific, Inc.) according to the manufacturer's protocol. Briefly, following siRNA transfection and DMSO or cisplatin treatment $(2 \mu \mathrm{M})$, A549rCDDP cells were collected by trypsinization (Invitrogen; Thermo Fisher Scientific, Inc.) and suspended in Annexin binding buffer (Invitrogen; Thermo Fisher Scientific, Inc.). Next, propidium iodide and Annexin V provided by Annexin-V/Dead Cell Apoptosis kit (Invitrogen; Thermo Fisher Scientific, Inc.) were added into the cell suspension and incubated at room temperature for $15 \mathrm{~min}$, followed by analysis using a BD FACSCalibur flow cytometer (BD Biosciences, Franklin Lakes, NJ, USA). The cell apoptosis rate was analyzed using FlowJo software (Version 10.4.1, FlowJo LLC, Ashland, OR, USA). The cells positive for Annexin-V staining were considered apoptotic cells.

Small interfering (si)RNA transfection. A total of 2 MALAT1 siRNAs (siRNA1 and siRNA2) and control siRNA were purchased from Invitrogen; Thermo Fisher Scientific, Inc. The siRNA sequences were as follows: MALAT1 siRNA1, 5'-GGGCUUCUCUUAACAUUUAtt-3'; MALAT1 siRNA2, 5'-GGGCAA AUAUUGGCAAUUAtt-3'. For the siRNA transfection, $2 \times 10^{6}$ A549 or A549rCDDP cells were seeded on 6 -well plates. The following day, $50 \mathrm{pM}$ siRNA were mixed with Lipofectamine ${ }^{\circledR}$ RNAiMAX (Invitrogen; Thermo Fisher Scientific, Inc.) in Opti-MEM (Thermo Fisher Scientific, Inc.) and incubated at $37^{\circ} \mathrm{C}$ for $15 \mathrm{~min}$. Subsequently, the siRNA1 or siRNA2 mixture was added into the culture medium of cells and cultured at $37^{\circ} \mathrm{C}$ for $24 \mathrm{~h}$ prior to cisplatin or vehicle treatment.

Construction of plasmid and transfection. Full-length MALAT1 was amplified from cDNA of 293 cells and cloned into a pcDNA3 plasmid (Addgene, Inc., Cambridge, MA, USA) using PrimeSTAR Max DNA Polymerase (Takara Bio, Inc.). The PCR thermocycling conditions were as follows: Denaturation at $98^{\circ} \mathrm{C}$ for $10 \mathrm{sec}$, annealing at $55^{\circ} \mathrm{C}$ for $10 \mathrm{sec}$ and elongation at $72^{\circ} \mathrm{C}$ for $10 \mathrm{sec}$, repeated for 35 cycles. For the overexpression of MALAT1, $5 \mu \mathrm{g}$ pcDNA3-MALTA1 plasmid was transfected into A549 cells using Lipofectamine ${ }^{\circledR}$ 2000 (Invitrogen; Thermo Fisher Scientific, Inc.), RT-PCR was used to confirm elevation of MALAT1 after $48 \mathrm{~h}$ according to the procedure above. The primer sequences for MALAT1 amplification were as follows: MALAT1 forward: GGCGGT ACCATGAAACAATTTGGAGAAG; MALAT1 reverse: GCGCTCGAGCTAAGTTTGTACATTTTGCC.

Luciferase reporter assay. The 3' untranslated region (UTR) of KLF4 was amplified from cDNA of 293 cells and inserted into pGL-3 (Promega Corporation, Madison, WI, USA) using PrimeSTAR Max DNA Polymerase (Takara Bio, Inc.). The PCR thermocycling conditions were as follows: denaturation at $98^{\circ} \mathrm{C}$ for $10 \mathrm{sec}$, annealing at $55^{\circ} \mathrm{C}$ for $10 \mathrm{sec}$ and elongation at $72^{\circ} \mathrm{C}$ for $10 \mathrm{sec}$, repeated for 35 cycles. Then, 293 cells were co-transfected with pGL3-KLF4 3'UTR WT, miR-145 mimics or miR-negative control mimics, in combination with a pcDNA3-MALAT1 plasmid or pcDNA3 and internal control pRL-TK plasmid using Lipofectamine 2000 (Invitrogen; Thermo Fisher Scientific, Inc.). The sequence of miR-145 mimics was 5'-GUCCAGUUUUCCCAGGAAUCC CU-3'. The sequence of miR-negative control mimics was

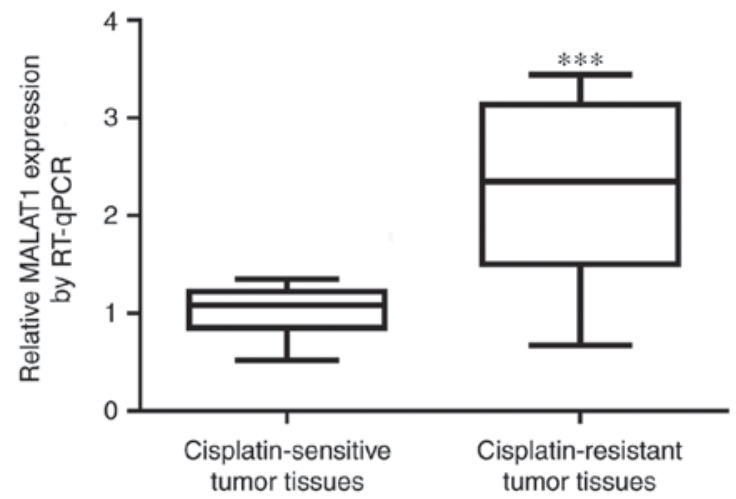

Figure 1. MALAT1 levels are increased in tumor tissues from patients with cisplatin-resistant NSCLC compared with those from patients with cisplatin-sensitive NSCLC. The different expression level of MALAT1 between 31 tumor tissues from patients with cisplatin-sensitive NSCLC and 21 tumor tissues from patients with cisplatin-resistant NSCLC was performed using RT-qPCR. ${ }^{* * *} \mathrm{P}<0.0001$. MALAT1, metastasis-associated lung adenocarcinoma transcript 1 ; NSCLC, non-small cell lung cancer; RT-qPCR, reverse transcription quantitative polymerase chain reaction.

5'-UCACAACCUCCUAGAAAGAGUAGA-3'. After $24 \mathrm{~h}$, luciferase activity and Renilla activity were measured using a Dual Luciferase Reporter Assay kit (Promega Corporation) according to manufacturer's protocol. Renilla activity was used as control.

Statistical analysis. All statistical analyses were performed using GraphPad Prism 5.0 software (GraphPad Software, Inc., La Jolla, CA, USA). The data were expressed as the mean \pm standard deviation. Differences between two groups were analyzed using an unpaired t-test. Differences from multiple groups were firstly analyzed using a one-way analysis of variance followed by Student-Newman-Keuls post-hoc analysis. $\mathrm{P}<0.05$ was considered to indicate a statistically significant difference. All experiments were repeated three times.

\section{Results}

MALAT1 levels are increased in tumor tissues from patients with cisplatin-resistant NSCLC compared with those from patients with cisplatin-sensitive NSCLC. To explore whether MALAT1 was involved in cisplatin resistance in NSCLC, MALAT1 levels from tumor tissues of 31 patients with cisplatin-sensitive NSCLC and 21 patients with cisplatin-resistant NSCLC were measured. Significant elevation of MALAT1 was detected in tumor tissues from patients with cisplatin-resistant NSCLC (Fig. 1). This suggested that the deregulation MALAT1 may contribute to cisplatin resistance in patients with NSCLC.

MALAT1 levels are associated with histological grades and metastasis. As summarized in Table I, there were 21 male patients and 31 female patients in the present study, with 32 patients aged $<60$ years old and 20 patients aged $\geq 60$ years old, 20 patients with well to intermediate differentiation and 32 patients with poor differentiation, and 41 patients with no metastasis and 11 patients with metastasis. 
Table I. Expression of MALAT1 in tissues from patients with non-small cell lung cancer.

\begin{tabular}{lcc}
\hline Clinicopathological factors & Cases, $n$ & MALAT, mean \pm SD \\
\hline Sex & & \\
Male & 21 & $1.51 \pm 0.21$ \\
Female & 31 & $1.48 \pm 0.23$ \\
Age, years & & $1.46 \pm 0.24$ \\
$<60$ & 32 & $1.52 \pm 0.23$ \\
$\geq 60$ & 20 & $1.10 \pm 0.32$ \\
Histological grade & & $1.93 \pm 0.58$ \\
Well to intermediate differentiation & 20 & \\
Poor differentiation & 32 & $1.16 \pm 0.38$ \\
Metastasis & & $1.84 \pm 0.47$ \\
No & 41 & $<0.01$ \\
Yes & 11 & $<0.01$ \\
\hline
\end{tabular}

SD, standard deviation; MALAT1, metastasis-associated lung adenocarcinoma transcript 1.

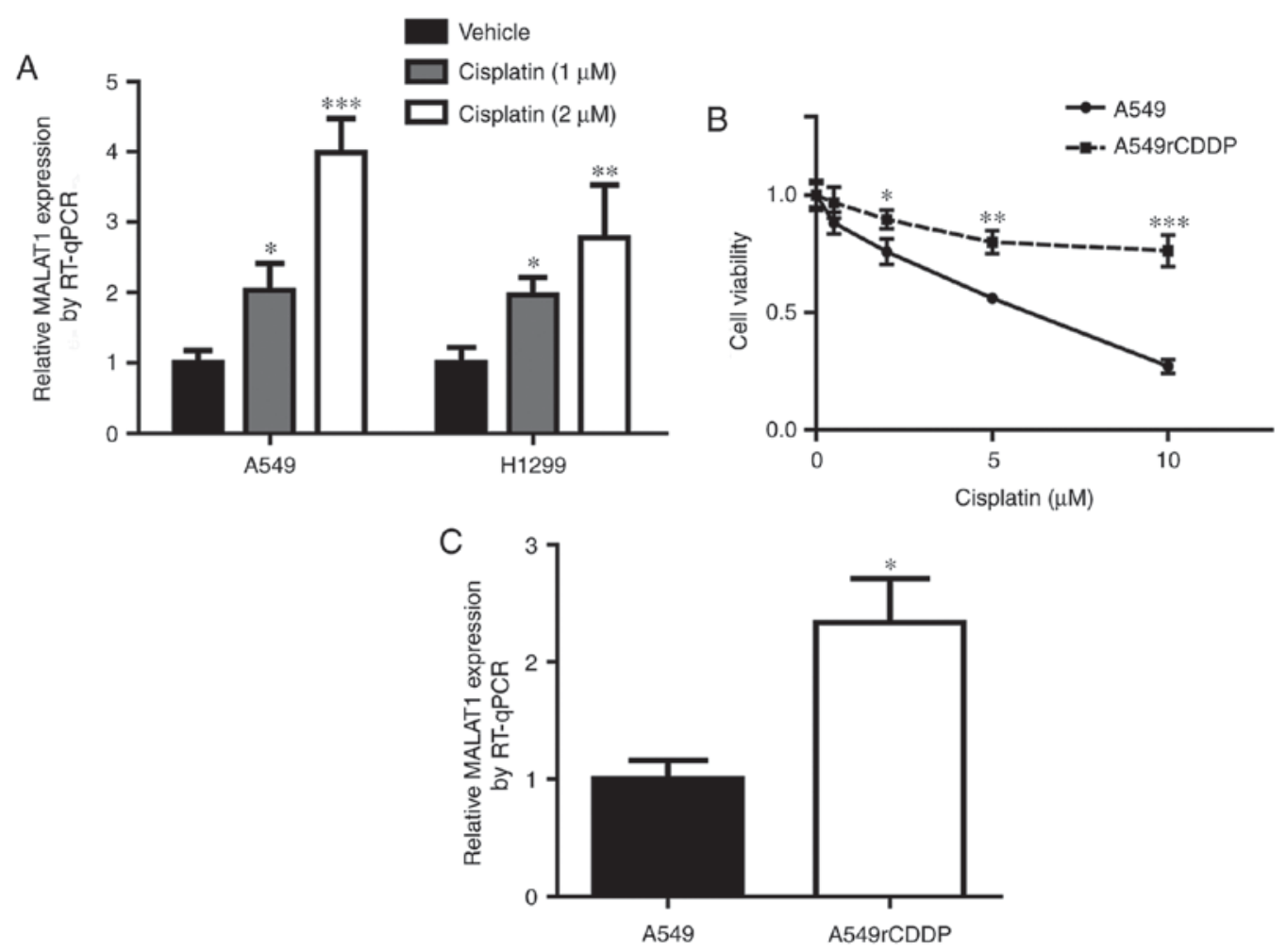

Figure 2. Increase of MALAT1 is observed in A549rCDDP cells and non-small cell lung cancer cells treated with cisplatin. (A) Increasing concentrations of cisplatin induced an increase in MALAT1 level in A549 cells and H1299 cells in a dose-dependent manner. Cisplatin $1 \mu \mathrm{M}$ vs. vehicle, Cisplatin $2 \mu \mathrm{M}$ vs. vehicle. (B) Compared with A549 cells, A549rCDDP cells were relatively insensitive towards cisplatin treatment. A549 vs. A549rCDDP. (C) Increased levels of MALAT1 mRNA were observed in A549rCDDP cells compared with A549 cells. ${ }^{*} \mathrm{P}<0.05,{ }^{* *} \mathrm{P}<0.001,{ }^{* * * *} \mathrm{P}<0.0001$. MALAT1, metastasis-associated lung adenocarcinoma transcript 1; RT-qPCR, reverse transcription quantitative polymerase chain reaction.

As for the difference of MALAT1 expression levels between male $(1.51 \pm 0.21)$ and female patients $(1.48 \pm 0.23)$ or patients aged $<60$ years old $(1.46 \pm 0.24)$ and $\geq 60$ years old $(1.52 \pm 0.23)$, there was no significant difference $(P=0.70$ and $P=0.35$, respectively). There was significant difference of MALAT1 expression levels $(\mathrm{P}<0.01$ and $\mathrm{P}<0.01$, respectively) between patients with well to intermediate differentiation $(1.10 \pm 0.32)$ and poor differentiation $(1.93 \pm 0.58)$, and between patients with metastasis $(1.84 \pm 0.47)$ or no metastasis $(1.16 \pm 0.38)$.

MALAT1 levels are increased in cisplatin-resistant NSCLC cells and NSCLC cells treated with cisplatin. To examine the role of MALAT1 during the development of cisplatin resistance, 2 NSCLC cell lines, A549 cells and H1299, were treated 

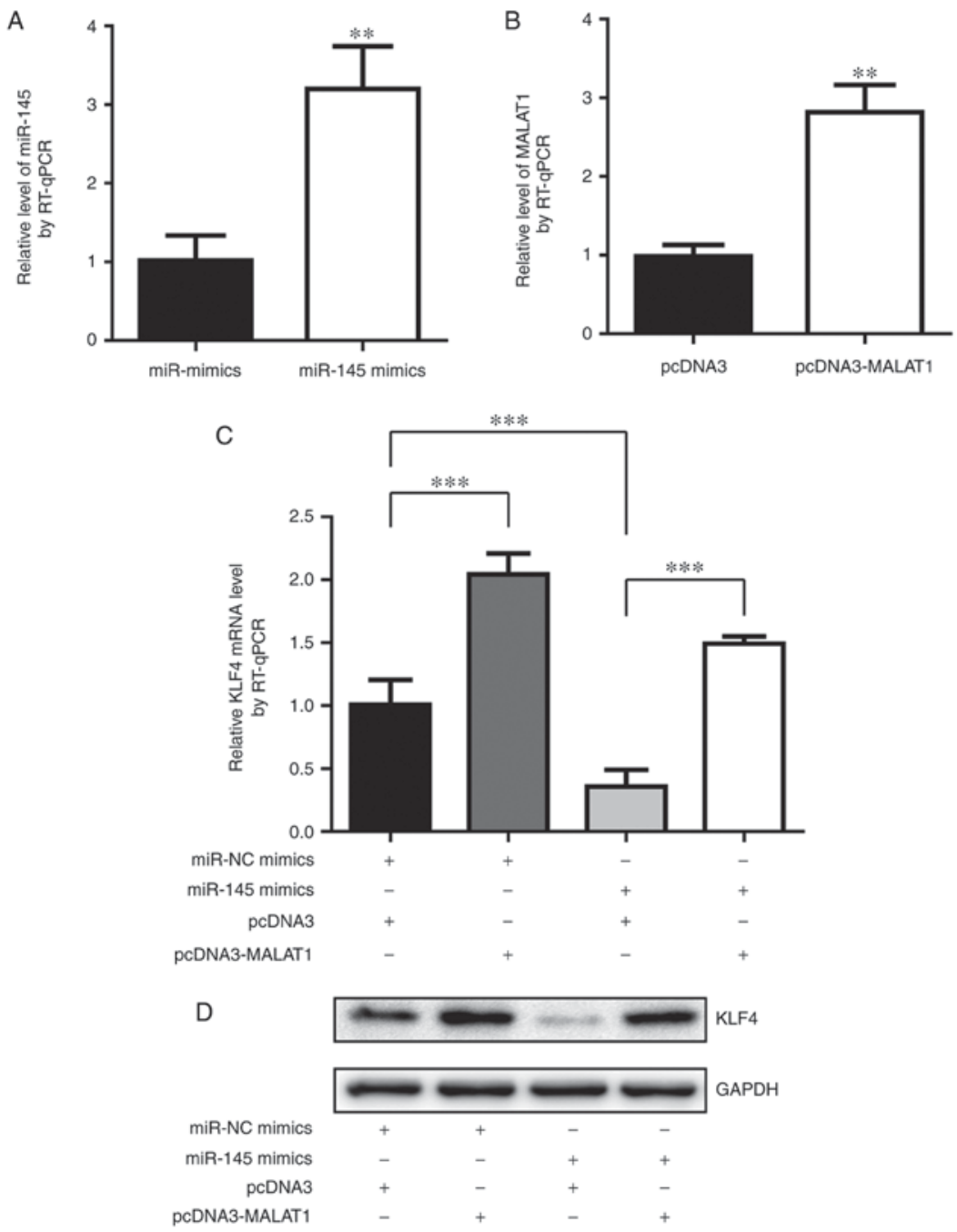

Figure 3. KLF4 is positively regulated by MALAT1 and negatively regulated by miR-145 in A549 cells. (A) miR-145 mimics markedly increased miR-145 levels in A549 cells. (B) MALAT1 levels were increased in A549 cells transfected with pcDNA3-MALAT1. (C) The KLF4 mRNA level was increased in response to transfection of pcDNA3-MALAT1 and was decreased following transfection of miR-145 mimics in A549 cells. (D) The protein level of KLF4 was increased in response to transfection of pcDNA3-MALAT1 and was decreased following transfection of miR-145 mimics in A549 cells. ** P<0.001 and ${ }^{* * *} \mathrm{P}<0.0001$. KLF4, Kruppel-like factor 4; MALAT1, metastasis-associated lung adenocarcinoma transcript 1; NSCLC, non-small cell lung cancer; RT-qPCR, reverse transcription quantitative polymerase chain reaction; $\mathrm{NC}$, negative control.

with ( 1 or $2 \mu \mathrm{M}$ of cisplatin. Notably, there was an elevation of MALAT1 level in response to cisplatin treatment in a dose-dependent manner (Fig. 2A). In addition, the present study aimed to analyze MALAT1 level in cisplatin-resistant cells. The cell viability assay indicated that A549rCDDP cells were relatively insensitive towards cisplatin treatment compared with the parental A549 cells (Fig. 2B). Indeed, the MALAT1 level was increased in A549rCDDP cells in comparison with A549 cells (Fig. 2C). These data implied that MALAT1 may contribute to cisplatin resistance in NSCLC cells.

MALAT1 regulated $K L F 4$, a chemotherapy resistance associated oncogene, in A549 cells. KLF4 is a transcription factor that controls stem cell reprogramming (17). Previously, KLF4 was identified to be associated with cisplatin resistance in several cancer types $(16,21)$. A previous study indicated that miR-145 may target KLF4 in bladder cancer cells (22). Notably, MALAT1 was suggested to repress miR-145 during endothelial to mesenchymal transition (23). To investigate the role of KLF4 and miR-145 in MALAT1-mediated

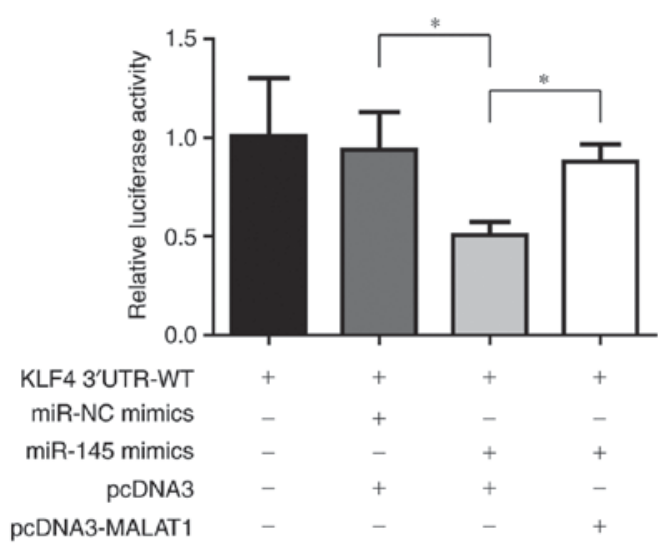

Figure 4. MALAT1 overexpression rescues miR-145-induced KLF4 transcription repression. The luciferase activity of 293 cells transfected with KLF4 3'UTR-WT was repressed following transfection with miR-145 mimics. Co-transfection of pcDNA3-MALAT1 and miR-145 mimics increased luciferase activity compared with transfection with miR-145 mimics alone. ${ }^{*} \mathrm{P}<0.05$. KLF4, Kruppel-like factor 4; MALAT1, metastasis-associated lung adenocarcinoma transcript 1; WT, wild-type; UTR, untranslated region; NC, negative control. 


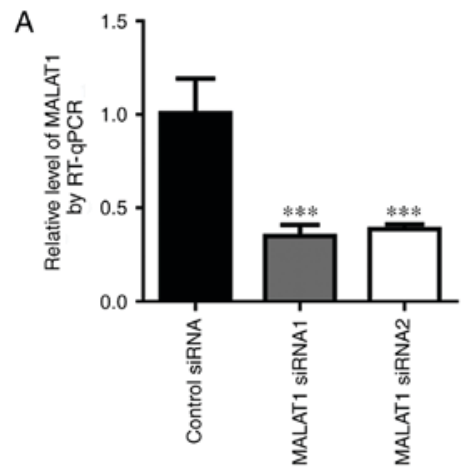

B

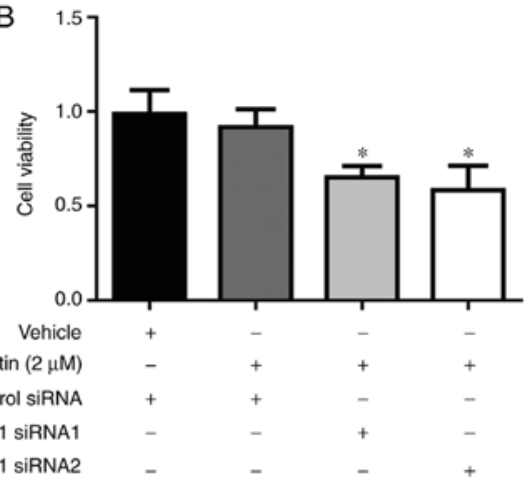

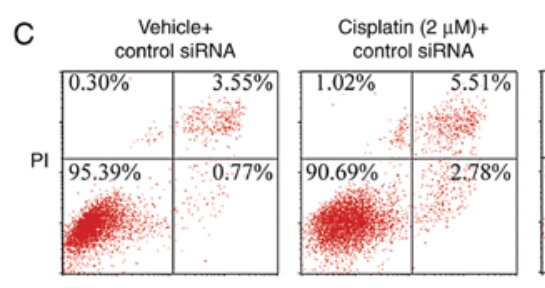
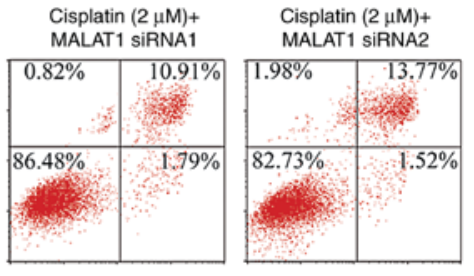

Annexin V

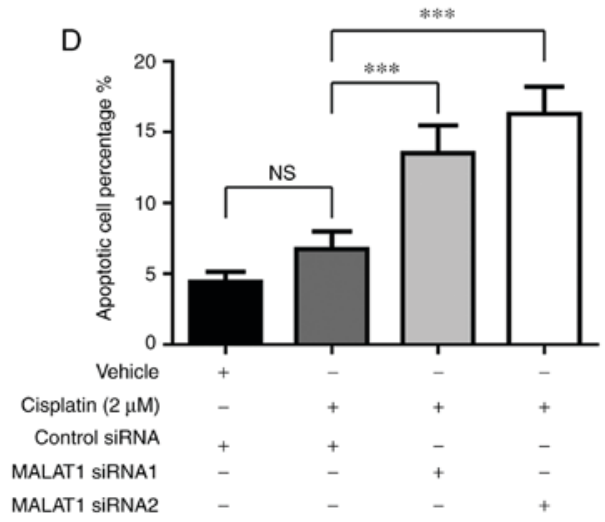

Figure 5. Silencing of MALAT1 reverses cisplatin resistance in A549rCDDP cells. (A) MALAT1 siRNA1 and siRNA2 decreased MALAT1 levels in A549rCDDP cells. MALAT1 siRNA1 vs. control siRNA, MALAT1 siRNA2 vs. control siRNA. (B) In A549rCDDP cells transfected with MALAT1 siRNA1 and siRNA2, cisplatin treatment for $48 \mathrm{~h}$ markedly suppressed cell proliferation. MALAT1 siRNA1 and cisplatin ( $2 \mu \mathrm{M})$ vs. control siRNA and cisplatin $(2 \mu \mathrm{M})$, MALAT1 siRNA2 and cisplatin $(2 \mu \mathrm{M})$ vs. control siRNA and cisplatin $(2 \mu \mathrm{M})$. (C) In MALAT1-silenced A549rCDDP cells, significantly increased cell apoptosis was detected following cisplatin treatment for $48 \mathrm{~h}$ compared with cells transfected with control siRNA. (D) Quantitative analysis of the flow cytometry data from C. (D) Annexin-V positive cells were considered apoptotic cells. ${ }^{*} \mathrm{P}<0.05$ and ${ }^{* * * *} \mathrm{P}<0.0001$. MALAT1, metastasis-associated lung adenocarcinoma transcript 1; si, small interfering; RT-qPCR, reverse transcription quantitative polymerase chain reaction; NS, not significant.

cisplatin resistance, the present study aimed to detect KLF4 level following MALAT1 or miR-145 overexpression. As demonstrated in Fig. 3A and B, miR-145 mimics and pcDNA-MALAT1 significantly increased miR-145 level and MALAT1 level in A549 cells, respectively. As hypothesized, the overexpression of MALAT1 markedly increased KLF4 mRNA level while miR-145 mimics decreased KLF4 mRNA level, and a combination of MALAT1 and miR-145 overexpression rescued miR-145-induced KLF4 downregulation (Fig. 3C). Consistently, MALAT1 overexpression increased KLF4 protein level compared with transfection of pcDNA3 and miR-145 mimics triggered a decrease in KLF4 protein level compared with cells transfected with miR-NC mimics (Fig. 3D). These data indicated that MALAT1 may function through the miR-145/KLF4 axis, to confer cisplatin resistance in NSCLC cells.

MALAT1 directly regulates miR-145 to control KLF4 levels. To confirm whether MALAT1 increased KLF4 level via targeting miR-145, a luciferase assay in 293 cells was performed. In cells transfected with KLF4 3'UTR-WT, it was identified that miR-145 mimics led to a decrease of luciferase activity and MALAT1 overexpression rescued this luciferase activity change (Fig. 4). This result indicated that KLF4 was regulated by MALAT1 via miR-145.

MALAT1 silencing reverses cisplatin resistance in A549rCDDP cells. The present study then aimed to confirm whether MALAT1 contributed to cisplatin resistance in NSCLC cells by siRNA-mediated gene silencing. As demonstrated in Fig. 5A, MALAT1 siRNA1 and siRNA2 significantly knocked down MALAT1 expression in A549rCDDP cells. Following MALAT1 silencing, cisplatin treatment induced a significant decrease in cell viability in A549rCDDP cells (Fig. 5B). Furthermore, compared with A549rCDDP cells treated with cisplatin, a significant increase in the rate of cell apoptosis was observed in MALAT1-silenced A549rCDDP cells following cisplatin treatment (Fig. 5C and D). These 
results directly indicated that the increase of MALAT1 contributed to cisplatin resistance in NSCLC.

\section{Discussion}

Cisplatin-based chemotherapy is a major therapeutic approach for the treatment of patients with NSCLC. However, although patients may respond to cisplatin at the initiation of the treatment, cisplatin exposure would induce a series of adaptive responses, leading to cisplatin resistance and tumor recurrence (24). The present study identified a MALAT1-miR-145-KLF4 axis was involved in driving cisplatin resistance in NSCLC.

MALAT1 was a well-characterized oncogene involved in the promotion of NSCLC cell proliferation and metastasis $(25,26)$. In the present study, it was also identified that MALAT1 levels were positively associated with histological grade and occurrence of metastasis. Recently, the upregulation of MALAT1 was demonstrated to increase Histone-lysine $\mathrm{N}$-methyltransferase EZH2 protein level and contribute to resistance towards oxaliplatin-based chemotherapy in patients with colorectal cancer (27). In the present study, cisplatin treatment of A549 cells greatly increased MALAT1 level. In addition, there was an elevation of MALAT1 level in A549rCDDP cells compared with the parental A549 cells. Importantly, the silencing of MALAT1 enhanced cisplatin-induced cell proliferation inhibition and cell apoptosis in A549rCDDP cells. These results collectively indicate that the abnormal expression of MALAT1 leads to cisplatin resistance in NSCLC.

Cancer stem cells are only a small proportion of the tumor cell population, and are crucial for tumor cell metastasis and drug resistance $(28,29)$. As a key transcription factor in regulating cell reprogramming and an important gene in the maintenance of cancer cell stemness $(30,31)$, KLF4 has been suggested to be associated with chemotherapy resistance in various types of cancer, including NSCLC $(18,32)$. A previous study identified that following benzo[a]pyrene treatment, an increase in KLF4 and MALAT1 protein levels was detected during the malignant transformation of the human bronchial epithelial BEAS-2B cell line (33). However, to the best of our knowledge, the regulatory association between KLF4 and MALTA1, and how their interplay contributed to cisplatin resistance has not yet been studied. KLF4 is a direct target of miR-145 (22). In the present study, in the A549 cells, it was identified that KLF4 was negatively regulated by miR-145 and positively regulated by MALAT1 at mRNA and protein levels. Using a luciferase assay, it was confirmed that MALAT1 indirectly regulated KLF4 at a transcription level via the repression of miR-145. These data indicated that MALTA1 may contribute to cisplatin resistance via regulating KLF4 level.

In conclusion, the data of the present study demonstrated that the MALAT1-miR-145-KLF4 axis functions as an important inducer of cisplatin resistance in NSCLC. Therefore, MALAT1 may be a promising predictor of cisplatin response and therapeutic target for patients with NSCLC.

\section{Acknowledgements}

Not applicable.

\section{Funding}

No funding was received.

\section{Availability of data and materials}

The datasets used and/or analyzed during the current study are available from the corresponding author on reasonable request.

\section{Authors' contributions}

YC designed the study, analyzed the data and wrote the manuscript. GL, XZ and FD collected the tissue samples, performed the experiments, and analyzed the data. RZ performed the experiments and analyzed the data.

\section{Ethics approval and consent to participate}

The present study was approved by the Ethics Committee of Shouguang People's Hospital. Each patient provided written consent to participate.

\section{Patient consent for publication}

Patients consented to the publication of their data.

\section{Competing interests}

The authors declare that they have no competing interests.

\section{References}

1. Molina JR, Yang P, Cassivi SD, Schild SE and Adjei AA: Non-small cell lun cancer: Epidemiology, risk factors, treatment, and survivorship. Mayo Clin Proc 83: 584-594, 2008.

2. Nishio K, Nakamura T, Koh Y, Suzuki T, Fukumoto H and Saijo N: Drug resistance in lung cancer. Curr Opin Oncol 11: 109-115, 1999.

3. Thum T: Noncoding RNAs and myocardial fibrosis. Nat Rev Cardiol 11: 655-663, 2014.

4. Kim C, Kang D, Lee EK and Lee JS: Long noncoding RNAs and RNA-binding proteins in oxidative stress, cellular senescence, and age-related diseases. Oxid Med Cell Longev 2017: 2062384, 2017.

5. Lin R, Maeda S, Liu C, Karin M and Edgington TS: A large noncoding RNA is a marker for murine hepatocellular carcinomas and a spectrum of human carcinomas. Oncogene 26: 851-858, 2007.

6. Fúri I, Kalmár A, Wichmann B, Spisák S, Schöller A, Barták B, Tulassay Z and Molnár B: Cell free DNA of tumor origin induces a 'Metastatic' expression profile in HT-29 cancer cell line. PLoS One 10: e0131699, 2015.

7. Jin Y, Feng SJ, Qiu S, Shao N and Zheng JH: LncRNA MALAT1 promotes proliferation and metastasis in epithelial ovarian cancer via the PI3K-AKT pathway. Eur Rev Med Pharmacol Sci 21: 3176-3184, 2017

8. Li H, Yuan X, Yan D, Li D1, Guan F, Dong Y, Wang H, Liu X and Yang B: Long non-coding RNA MALAT1 decreases the sensitivity of resistant glioblastoma cell lines to temozolomide. Cell Physiol Biochem 42: 1192-1201, 2017.

9. Larne O,Hagman Z,Lilja H, Bjartell A, Edsjö A and Ceder Y: miR-145 suppress the androgen receptor in prostate cancer cells and correlates to prostate cancer prognosis. Carcinogenesis 36: 858-866, 2015.

10. Chang S, Gao L, Yang Y, Tong D, Guo B, Liu L, Li Z, Song T and Huang C: miR-145 mediates the antiproliferative and gene regulatory effects of vitamin D3 by directly targeting E2F3 in gastric cancer cells. Oncotarget 6: 7675-7685, 2015.

11. Ju BL, Chen YB, Zhang WY, Yu CH, Zhu DQ and Jin J: miR-145 regulates chemoresistance in hepatocellular carcinoma via epithelial mesenchymal transition. Cell Mol Biol (Noisy-le-grand) 61: 12-16, 2015. 
12. Ye Z, Shen N, Weng Y, Li K, Hu L, Liao H, An J, Liu L, Lao S and Cai S: Low miR-145 silenced by DNA methylation promotes NSCLC cell proliferation, migration and invasion by targeting mucin 1. Cancer Biol Ther 16: 1071-1079, 2015.

13. Lopez-Ayllon BD, Moncho-Amor V, Abarrategi A, Ibañez de Cáceres I, Castro-Carpeño J, Belda-Iniesta C, Perona R and Sastre L: Cancer stem cells and cisplatin-resistant cells isolated from non-small-lung cancer cell lines constitute related cell populations. Cancer Med 3: 1099-1111, 2014.

14. Chen YJ, Wu CY, Chang CC, Ma CJ, Li MC and Chen CM: Nuclear Krüppel-like factor 4 expression is associated with human skin squamous cell carcinoma progression and metastasis. Cancer Biol Ther 7: 777-782, 2008.

15. Shi M, Cui J, Du J, Wei D, Jia Z, Zhang J, Zhu Z, Gao Y and $\mathrm{X}$ ie $\mathrm{K}$ : A novel KLF4/LDHA signaling pathway regulates aerobic glycolysis in and progression of pancreatic cancer. Clin Cancer Res 20: 4370-4380, 2014.

16. Huang J, Liu K, Song D, Ding M, Wang J, Jin Q and Ni J: Krüppel-like factor 4 promotes high-mobility group box 1 -induced chemotherapy resistance in osteosarcoma cells Cancer Sci 107: 242-249, 2016.

17. Fadous-Khalifé MC, Aloulou N, Jalbout M, Hadchity J, Aftimos G, Paris F and Hadchity E: Krüppel-like factor 4: A new potential biomarker of lung cancer. Mol Clin Oncol 5: 35-40, 2016.

18. Liu MY, Li XQ, Gao TH, Cui Y, Ma N2, Zhou Y2 and Zhang GJ: Elevated HOTAIR expression associated with cisplatin resistance in non-small cell lung cancer patients. J Thorac Dis 8: 3314-3322, 2016

19. Petersen I and Warth A: Lung cancer: Developments, concepts, and specific aspects of the new WHO classification. J Cancer Res Clin Oncol 142: 895-904, 2016.

20. Livak KJ and Schmittgen TD: Analysis of relative gene expression data using real-time quantitative PCR and the 2(-Delta Delta C(T)) method. Methods 25: 402-408, 2001

21. Jia Y, Zhang W, Liu H, Peng L, Yang Z and Lou J: Inhibition of glutathione synthesis reverses Krüppel-like factor 4-mediated cisplatin resistance. Cancer Chemother Pharmacol 69: 377-385, 2012.

22. Minami K, Taniguchi K, Sugito N, Kuranaga Y, Inamoto T, Takahara K, Takai T, Yoshikawa Y, Kiyama S, Akao Y and Azuma H: MiR-145 negatively regulates Warburg effect by silencing KLF4 and PTBP1 in bladder cancer cells. Oncotarget 8: 33064-33077, 2017.

23. Xiang Y, Zhang Y, Tang Y and Li Q: MALAT1 modulates TGF- $\beta$ 1-induced endothelial-to-mesenchymal transition through downregulation of miR-145. Cell Physiol Biochem 42: 357-372, 2017.

24. Galluzzi L, Vitale I, Michels J, Brenner C, Szabadkai G, Harel-Bellan A, Castedo M and Kroemer G: Systems biology of cisplatin resistance: Past, present and future. Cell Death Dis 5: e1257, 2014.
25. Guo F, Guo L, Li Y, Zhou Q and Li Z: MALAT1 is an oncogenic long non-coding RNA associated with tumor invasion in non-small cell lung cancer regulated by DNA methylation. Int J Clin Exp Pathol 8: 15903-15910, 2015.

26. Guo F, Jiao F, Song Z, Li S, Liu B, Yang H, Zhou Q and Li Z: Regulation of MALAT1 expression by TDP43 controls the migration and invasion of non-small cell lung cancer cells in vitro. Biochem Biophys Res Commun 465: 293-298, 2015.

27. Li P, Zhang X, Wang H, Wang L, Liu T, Du L, Yang Y and Wang C: MALAT1 is associated with poor response to oxaliplatin-based chemotherapy in colorectal cancer patients and promotes chemoresistance through EZH2. Mol Cancer Ther 16: 739-751, 2017.

28. Farnie G, Sotgia F and Lisanti MP: High mitochondrial mass identifies a sub-population of stem-like cancer cells that are chemo-resistant. Oncotarget 6: 30472-30486, 2015.

29. Huang Z, Wu T, Liu AY and Ouyang G: Differentiation and transdifferentiation potentials of cancer stem cells. Oncotarget 6: 39550-39563, 2015.

30. Yu S, Cai X, Wu C, Wu L, Wang Y, Liu Y, Yu Z, Qin S, Ma F, Thiery JP and Chen L: Adhesion glycoprotein CD44 functions as an upstream regulator of a network connecting ERK, AKT and Hippo-YAP pathways in cancer progression. Oncotarget 6: 2951-2965, 2015.

31. Li Y, Xian M, Yang B, Ying M and He Q: Inhibition of KLF4 by statins reverses adriamycin-induced metastasis and cancer stemness in osteosarcoma cells. Stem Cell Reports 8: 1617-1629, 2017.

32. Lund RJ, Huhtinen K, Salmi J, Rantala J, Nguyen EV, Moulder R, Goodlett DR, Lahesmaa R and Carpén O: DNA methylation and transcriptome changes associated with cisplatin resistance in ovarian cancer. Sci Rep 7: 1469, 2017.

33. Liu Y, Lu R, Gu J, Chen Y, Zhang X, Zhang L, Wu H, Hua W and Zeng J: Aldehyde dehydrogenase $1 \mathrm{~A} 1 \mathrm{up}$-regulates stem cell markers in benzo[a]pyrene-induced malignant transformation of BEAS-2B cells. Environ Toxicol Pharmacol 45: 241-250, 2016.

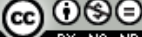

This work is licensed under a Creative Commons Attribution-NonCommercial-NoDerivatives 4.0 International (CC BY-NC-ND 4.0) License. 